\title{
Cyclones dans des bulles de savon
}

Hamid Kellay (hamid.kellay@u-bordeaux.fr)

Laboratoire Ondes et Matière d’Aquitaine (LOMA), UMR 5798 CNRS/Université de Bordeaux,

351 cours de la Libération, 33405 Talence Cedex

Une bulle de savon chauffée

à son équateur peut être

le théâtre d'une agitation

importante, avec l'apparition

à sa surface de différentes

structures : des panaches

thermiques donnant naissance

à un écoulement turbulent,

et des tourbillons solitaires

avec une dynamique propre.

Nos expériences se sont

focalisées sur les propriétés

à la fois de cette turbulence,

mais aussi de ces tourbillons

solitaires.

Malgré la simplicité de ces

objets, les résultats qu'on

obtient sont parfois étonnants,

notamment par leur analogie

avec les cyclones terrestres.

Remerciements

L'auteur remercie F. Seychelles, F. Ingremeau, T. Meuel, M. Bessafi, C. H. Bruneau, P. Fischer et G. Prado pour leurs contributions à ces recherches, ainsi que E. Maillard et S. Bosio pour leurs réalisations des montages expérimentaux. Ce travail a bénéficié d'un financement de l'ANR pour le projet «Cyclobulle ».

\section{Apprivoiser la bulle}

Mettez un peu d'eau savonneuse dans une assiette, prenez une paille et soufflez un peu d'air dans cette eau. Le résultat est intuitif : des bulles de savon, ou plutôt des demi-bulles de savon, se forment et flottent sur l'eau. Si l'eau est assez chaude $\left(50{ }^{\circ} \mathrm{C}\right.$ par exemple) et si vous observez la surface des bulles attentivement, vous remarquerez que des mouvements animent cette surface.

Partant de cette simple observation, nous avons réalisé au laboratoire des montages expérimentaux, dont la géométrie et le chauffage sont plus contrôlés (fig. 1). L'eau savonneuse est versée dans une rainure gravée dans un disque de laiton, et chauffée à la bonne température. Une pipette fixée à un tube flexible est utilisée pour souffler la demi-bulle de savon. La température de l'air ambiant autour de la bulle est contrôlée, permettant l'établissement d'un gradient de température sur la surface de la bulle entre l'équateur et le pôle. Ce gradient est mesuré soit avec un thermocouple, soit avec une caméra infrarouge.

Le fait de chauffer le socle sur lequel la demi-bulle repose favorise les mouvements à la surface de cette pellicule très fine (de quelques micromètres d'épaisseur), constituée d'un film d'eau et de tensioactifs. L'origine de ces mouvements est liée aux variations de la tension superficielle de la solution de savon avec la température, mais surtout à la dilatation thermique de cette solution qui est l'effet dominant [1]. Cette agitation peut être plus ou moins intense en fonction de la différence de température entre l'équateur et le pôle.

Pour voir ce qui se passe à la surface, nous utilisons une caméra couleur et une source de lumière blanche avec un écran diffuseur pour éclairer la bulle et faire ressortir les couleurs d'interférences

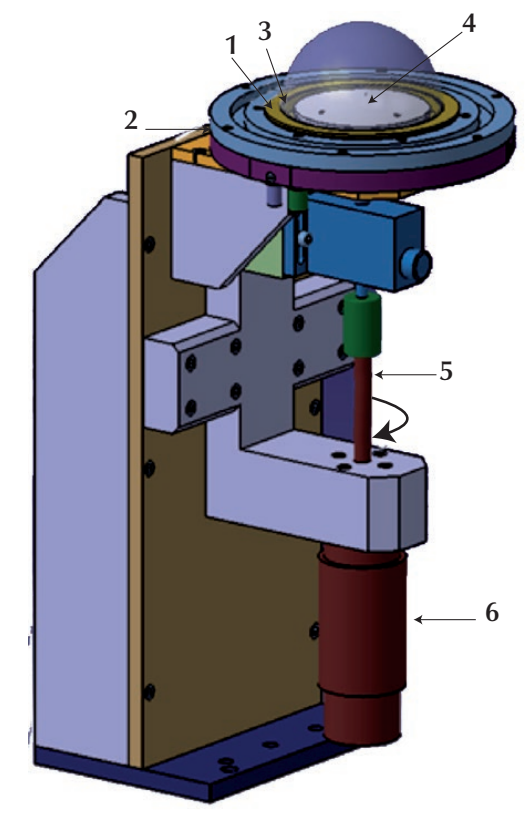

1. Montage expérimental. Un disque en laiton (1) avec une rainure circulaire (3) peut tourner en utilisant un moteur (6) relié par un arbre (5). Ce disque est chauffé par la proximité d'un creux en forme d'anneau (2) relié à un bain à circulation d'eau. La bulle est soufflée à l'aide d'une pipette à travers la solution de savon dans la rainure (3). La face interne du disque de laiton est recouverte d'un revêtement de téflon de $2 \mathrm{~mm}$ d'épaisseur (4), afin de minimiser l'échauffement de l'air à l'intérieur de la bulle. La température à l'équateur de la bulle est régulée par la température du bain d'eau.

correspondant à des variations locales de l'épaisseur du film de savon et grâce auxquelles les écoulements à la surface de la bulle peuvent être observés (voir dans la référence [2] d'autres expériences utilisant les films de savon comme milieu bidimensionnel pour étudier les écoulements). La figure 2 montre les panaches thermiques émanant de l'équateur de la bulle : plus chauds et moins denses que les régions environnantes, ils s'élèvent vers le pôle. Ces panaches ou "plumes thermiques" sont essentiels pour transporter la chaleur 

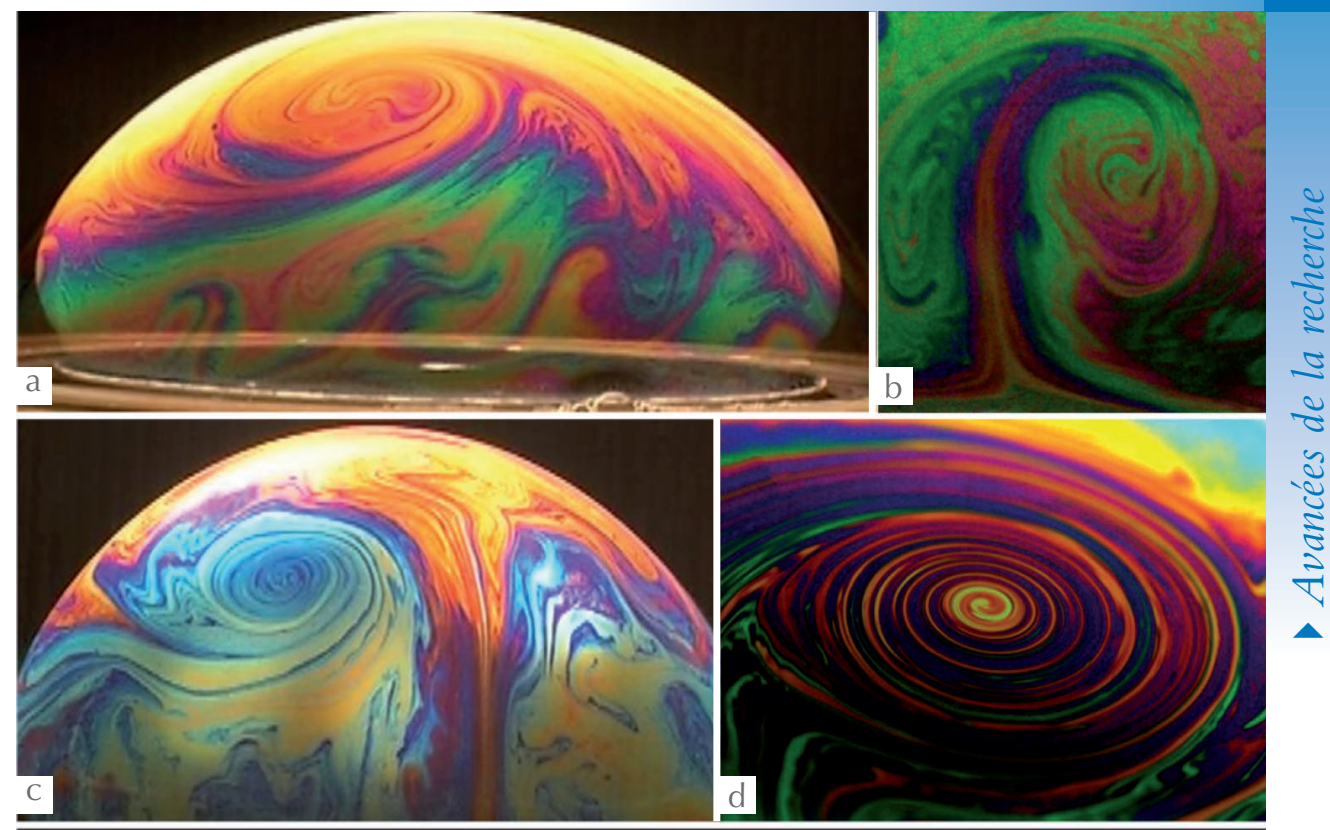

des régions chaudes à l'équateur vers les régions plus froides au pôle. Cette convection thermique est très répandue dans plusieurs contextes industriels et naturels, allant d'une casserole d'eau bouillante à la dynamique du manteau terrestre ; elle a suscité et continue de susciter l'intérêt des scientifiques et des ingénieurs.

\section{Que nous raconte la bulle?}

Dans notre contexte, la bulle est une cellule de convection non conventionnelle et sans murs latéraux, contrairement à la majorité des expériences effectuées jusqu'à présent. De plus, en augmentant le gradient de température au-delà d'un certain seuil, l'écoulement sur la surface de la bulle devient turbulent et des tests des lois d'échelle régissant la convection turbulente peuvent être effectués, à la fois pour les fluctuations de vitesse et pour les fluctuations de température [3]. Ce type de tests reste difficile à explorer dans les cellules conventionnelles, notamment à cause des murs latéraux.

Sur la bulle, les plumes et la convection thermique sont accompagnées de temps en temps de tourbillons solitaires qui émergent de cette agitation. Ces tourbillons se forment généralement quand un grand panache, résultant de la fusion de quelques plumes plus petites, s'élève à proximité du sommet de la bulle (photographie c de la figure 2). Une fois formés, les tourbillons peuvent être suivis en utilisant l'imagerie vidéo. Un tourbillon solitaire de ce genre est visible sur la figure $2 \mathrm{~d}$. On notera qu'il a un centre bien défini et se développe comme une structure en spirale, de dimensions comprises entre 1 et $2 \mathrm{~cm}$. Ces tourbillons simples peuvent également être obtenus dans les simulations numériques

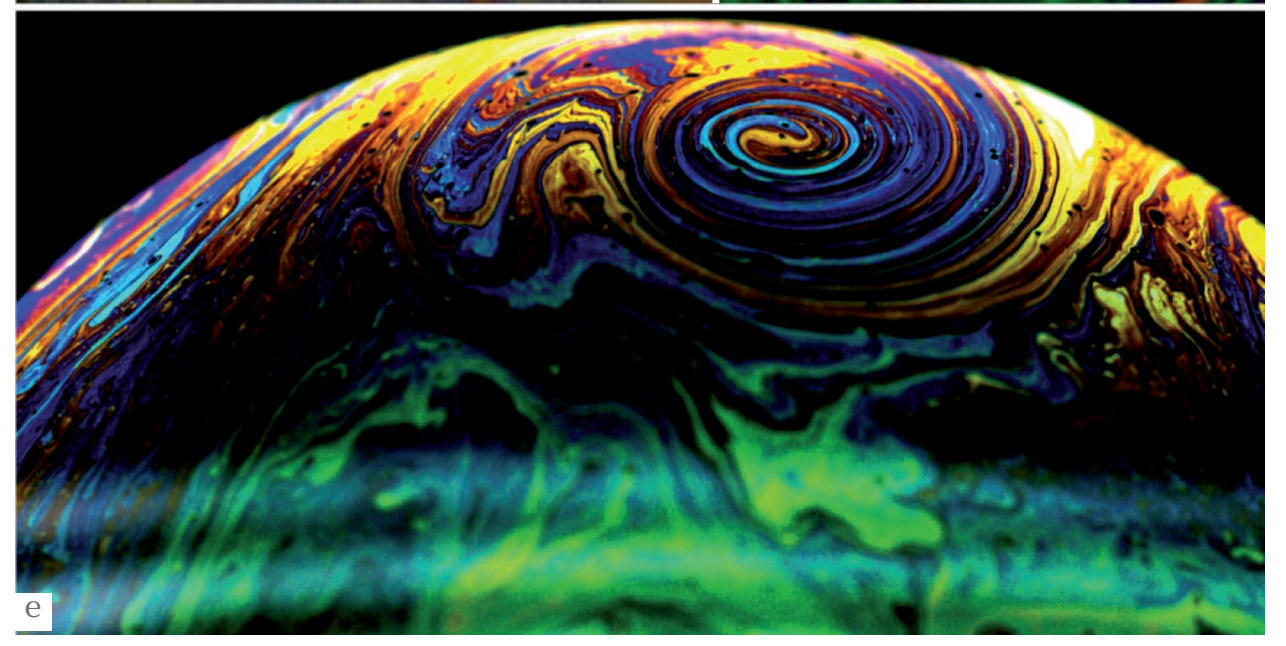

2. Photos de panaches et tourbillons observés dans des bulles de savon soumises à un gradient de température. Les images (a) et (b) montrent le détachement de panaches thermiques à proximité de l'équateur. L'image (c) montre la formation d'un tourbillon à partir d'un panache géant qui lui-même est le résultat de la fusion de plusieurs panaches de petite taille. L'image (d) montre un zoom sur un tourbillon solitaire. L'image (e) montre la bulle complète avec un tourbillon proche du pôle. Les couleurs sont des couleurs d'interférences de lumière blanche réfléchie par la mince couche d'eau, de quelques micromètres d'épaisseur, constituant la bulle. Les différentes couleurs correspondent à des épaisseurs différentes du film d'eau savonneuse. Le diamètre typique des bulles est de $12 \mathrm{~cm}$.

de la convection thermique à deux dimensions sur une surface sphérique, de sorte qu'ils ne sont pas particuliers à des bulles de savon [3].

Des tourbillons solitaires tels que ceux observés ici ne sont pas très communs dans les écoulements de fluides. En général, les tourbillons viennent en paires comme dans le sillage derrière un obstacle, où une allée dite de Bénard-von Karman est produite avec des paires de tourbillons se détachant régulièrement derrière l'obstacle. Des tourbillons solitaires peuvent par contre être observés à la surface de certaines planètes et suscitent dans la communauté scientifique un débat sur leur durée de vie (comme pour la tache rouge de Jupiter), leur structure ou leur emplacement. La nature quasi bidimensionnelle des écoulements atmosphériques à grandes échelles a été évoquée comme une des raisons de la stabilité de ces structures. Dans notre cas, les raisons exactes de la formation et la présence de ces tourbillons solitaires de longue durée de vie (plusieurs secondes) ne sont pas claires, mais rappelons que le système est quasi bidimensionnel vu la faible épaisseur du film de savon. En outre, dans une cellule de convection classique, une circulation globale et persistante peut avoir lieu. Il se peut que dans notre cellule sans murs, ces tourbillons soient les analogues ou les reliquats de cette circulation globale.

Notre système permet par contre d'étudier en détail les propriétés de ces structures. L'écoulement a lieu dans une couche très mince comparée à la taille de la bulle, la courbure est naturellement présente 
>>

(la bulle est sphérique), et une rotation peut être ajoutée pour se rapprocher le plus possible d'un modèle de laboratoire des écoulements atmosphériques. À cet effet, le montage expérimental a été modifié de telle sorte que des fréquences de rotation allant jusqu'à deux tours par seconde puissent être imposées (voir schéma du montage dans la figure 1). L'effet de la rotation de la bulle se révèle non trivial, car cette rotation inhibe la présence de tourbillons de longue durée de vie et les confine à des régions proches du pôle $[4,5]$. Tandis que le sens de rotation de ces tourbillons peut être horaire ou antihoraire avec une probabilité égale pour le cas sans rotation, la rotation de la bulle fixe le sens de rotation des tourbillons qui sont majoritairement cycloniques, c'est-à-dire qu'ils tournent dans le même sens que la bulle.

Une question importante qui peut être traitée en détail dans nos expériences concerne le champ de vitesse autour du tourbillon et sa variation en fonction de la distance au centre et du temps. Les mesures peuvent être réalisées sur de longues périodes (plusieurs fois le temps nécessaire pour faire un tour complet autour du centre du tourbillon, qui est de l'ordre de $0,1 \mathrm{~s}$ ), car ces tourbillons peuvent persister pendant plusieurs dizaines de secondes. Elles sont effectuées par le suivi temporel d'inhomogénéités d'épaisseur de la bulle autour du tourbillon, permettant des mesures à différentes distances du centre (dans le référentiel du centre du tourbillon). L'analyse des champs de vitesses déterminés par corrélation d'images ou par suivi d'inhomogénéités d'épaisseur permet d'obtenir à la fois la vitesse et la vorticité (le rotationnel du vecteur vitesse, indiquant un taux de rotation local) autour du tourbillon. Alors que la vorticité est maximale au centre du tourbillon, la vitesse augmente, passe par un maximum et finit par décroître au fur et à mesure qu'on s'éloigne du centre. La dépendance trouvée est bien connue : c'est un tourbillon gaussien, dont la vitesse à grande distance $(r)$ du centre décroît en $\exp \left(-\mathrm{r}^{2}\right)$; cela permet de décrire le champ de vitesse des tourbillons dans les écoulements peu profonds, et ressemble à l'expression utilisée pour modéliser les cyclones terrestres.

Un exemple de variation temporelle de la vitesse, dans le référentiel du centre du tourbillon et à une distance fixe de ce centre, est illustré dans la figure 3 .

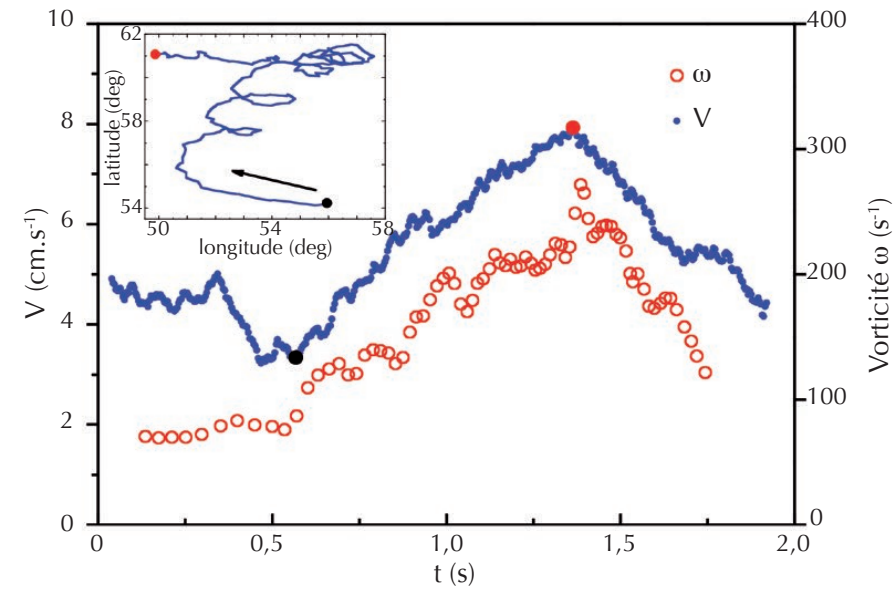

3. Dynamique à temps longs de la vitesse tangentielle (ronds bleus pleins, axe de gauche) et de la vorticité (ronds rouges vides, axe de droite) autour du centre d'un tourbillon solitaire. La mesure de la vitesse est faite à une distance fixe $(6 \mathrm{~mm})$ du centre du tourbillon. L'insert montre la trajectoire du centre du tourbillon en latitude et longitude, et son mouvement trochoïdal, visible pendant la phase d'augmentation de vitesse (intensification) située entre les points noir et rouge.
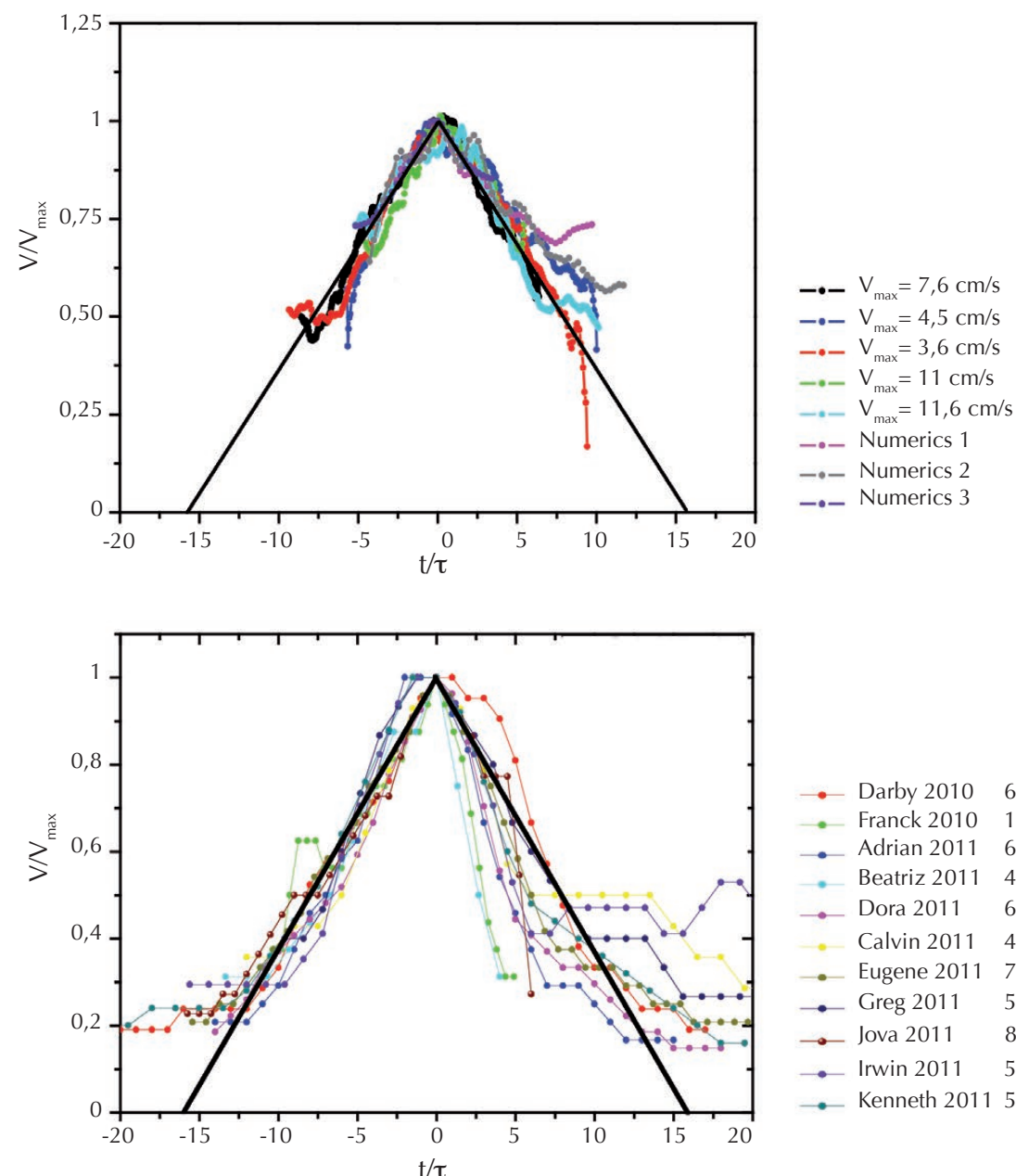

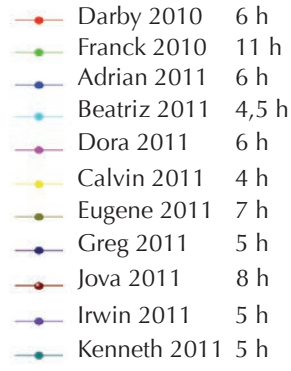

4. Comparaison des dynamiques d'intensification et de déclin des tourbillons en expérience (bulles), simulation numérique et conditions réelles (ouragans). La vitesse à une distance fixe du centre du tourbillon est normalisée par la vitesse maximale, et le temps, normalisé par un temps caractéristique $\tau$, est déplacé de sorte que la position de la vitesse maximale est à zéro. (a) Cinq événements d'intensification différents pour des fréquences de rotation de la bulle de 0,2 ou 0,6 Hz, et trois événements d'intensification de tourbillons de simulations numériques. (b) Intensification de plusieurs ouragans dans le Pacifique Nord-Est, identifiés à droite de la figure par leurs noms et leurs temps caractéristiques. 
Cette variation temporelle montre une période de faible intensité (faible vitesse) suivie d'une période d'augmentation de la vitesse (dite "intensification »), puis d'une période de déclin. Le tourbillon suivi ici finit par réduire sa vitesse et, à la fin, disparaît pratiquement dans l'écoulement environnant.

Pendant la période d'intensification, la trajectoire du centre du tourbillon montre un mouvement particulier connu sous le nom de mouvement trochoidal : comme on le voit dans l'insert de la figure 3, le centre $\mathrm{du}$ tourbillon vacille à quelques reprises autour de sa position moyenne. La période d'oscillation du centre est à peu près un temps de révolution (de l'ordre de 0,1 s). Ce type de mouvement du centre du tourbillon, circulaire ou elliptique superposé à une dérive, a été observé et documenté pour certains cyclones tropicaux et a donné lieu à beaucoup de débats sur son origine et son lien avec l'intensité (vitesse) du tourbillon. Dans notre cas, ce lien n'est pas clair, car ce mouvement trochoïdal a été observé à la fois pour la phase d'intensification comme pour l'exemple cité plus haut, mais aussi pendant la phase de déclin pour d'autres tourbillons. Différentes raisons ont été proposées pour expliquer ces oscillations : l'instabilité de l'œil du tourbillon ou l'existence d'un double œil. Nos mesures de vitesse semblent exclure l'hypothèse du double œil, car nos visualisations ne semblent pas indiquer de grandes déformations du centre du tourbillon au cours de cette phase.

Revenons maintenant à la variation de la vitesse de rotation des tourbillons en fonction du temps. Dans la figure 4a, plusieurs événements d'intensification des tourbillons sur la bulle de savon ainsi que de tourbillons issus de nos simulations numériques sont présentés. Notez que les deux ensembles de données montrent une période d'intensification où la vitesse augmente jusqu'à une valeur maximale, notée $V_{\max }$, suivie d'une baisse de l'intensité. L'échelle de temps dans ce graphique a été translatée, de sorte que $\mathrm{V}_{\max }$ se produit à temps zéro, et a été normalisée par un temps caractéristique $\tau$. La vitesse a été normalisée par $\mathrm{V}_{\max }$. Une caractéristique surprenante de ce graphique est que par un simple changement d'échelle de la vitesse et du temps, toutes nos données se superposent sur une seule courbe. Cette superposition indique que la dynamique de l'intensification présente des caractéristiques similaires pour différents tourbillons à différentes fréquences de rotation de la bulle, ainsi que pour les tourbillons issus des simulations numériques. Le temps caractéristique $\tau$ utilisé pour adimensionner les données varie d'un tourbillon à un autre, mais se situe autour d'une valeur moyenne d'environ $0,07 \mathrm{~s}$, aussi bien pour les tourbillons issus de la bulle de savon que dans les simulations numériques. Ce temps est comparable au temps de révolution de ces tourbillons $(\sim 0,1 \mathrm{~s})$. Alors que le temps $\tau$ semble être bien défini, la vitesse maximale atteinte par le tourbillon ne semble pas suivre de règle particulière et des vitesses différentes ont été observées, allant d'environ 3 à $12 \mathrm{~cm} / \mathrm{s}$.

La dynamique à temps longs de ces tourbillons semble donc bien décrite par une relation simple : $\mathrm{V}(\mathrm{t})=\mathrm{V}_{\max }(1-|\mathrm{t}| / 16 \tau)$, où la vitesse augmente linéairement avec le temps avant le maximum et décroît linéairement après le maximum. Cette relation peut être réécrite comme $\mathrm{dV} / \mathrm{dt}= \pm \mathrm{V}_{\max } / 16 \tau$, indiquant que le taux $\mathrm{dV} / \mathrm{dt}$ d'intensification ou de déclin des tourbillons est lié simplement à la vitesse maximale que le tourbillon peut atteindre, et au temps $\tau$. Si ce dernier est lié au temps de révolution de ces tourbillons comme le montrent nos expériences, la vitesse maximale que peut atteindre le tourbillon est codée dans les taux d'intensification et de déclin, i.e. $\mathrm{dV} / \mathrm{dt}$. Pour l'instant, nous n'avons pas d'explication de cette relation. De plus, nous ne connaissons pas d'autres expériences où les vitesses de rotation de tourbillons isolés sont mesurées sur des temps longs et dont la dynamique puisse être comparée à celle déduite de nos mesures.

\section{Osons l'analogie}

Un cas où cette dynamique à temps long est bien documentée est celui des cyclones tropicaux. Ces tourbillons géants ont aussi un cycle d'intensification et de déclin qui rappelle ce que nous avons observé sur les bulles. Tout en gardant à l'esprit les grandes différences dans les mécanismes ainsi que dans l'énergétique des tourbillons de savon et des cyclones tropicaux, la question de savoir si ces similitudes qualitatives (mouvement trochoïdal, intensification et déclin) peuvent être rendues plus quantitatives a été examinée.

Dans la figure $4 \mathrm{~b}$, nous montrons la variation de la vitesse des vents $\mathrm{V}(\mathrm{t})$ en fonction $d u$ temps $t$ pour quelques cyclones tropicaux. Notez que cette figure utilise la même normalisation que la figure 4a pour superposer les données. La figure $4 \mathrm{~b}$ montre que cette normalisation fonctionne raisonnablement bien pour l'ensemble des données avec des constantes de temps $\tau$ qui varient, mais qui tournent autour de 6 heures. Cette superposition des données montre qu'elles obéissent à la même règle de variation que celle trouvée pour nos tourbillons (en bulles de savon ou numériques), sauf que la constante de temps est propre à chaque système : autour de 6 heures pour les cyclones (une analyse de 171 cyclones suggère que $\tau$ a une moyenne de 6 heures avec un écart type d'environ 2 heures [4]) et autour de 0,07 seconde pour nos tourbillons. Chaque système semble donc être caractérisé par une constante de temps particulière. La superposition des données proposée ici indique que la dynamique d'intensification des tourbillons peut avoir un caractère générique indépendant de la nature exacte du tourbillon. Un tel caractère peut s'avérer très utile [6] : connaissant la constante de temps et le taux d'intensification initial, l'intensité (vitesse des vents) maximale que le cyclone ou le tourbillon peut atteindre, ainsi que le moment où elle sera atteinte, peuvent être estimés.

Ainsi, les objets du quotidien peuvent enfermer quelques secrets qu' on peut extraire si on a la patience de les apprivoiser et de les observer. Les mouvements dans la bulle de savon ont une analogie avec des phénomènes naturels trois cent mille fois plus lents, analogie qui se révèle bien plus féconde qu'on aurait pu l'imaginer au départ.

\section{En savoir plus}

1• B. Martin et X.L. Wu, Phys. Rev. Lett., 80 (1998) 1891.

$2 \bullet Y$. Couder, « Les tourbillons de l'atmosphère », Science et Vie, n¹61 (1987) 128-133.

3• F. Seychelles et al., Phys. Rev. Lett. 105 (2010) 264502.

4 T. Meuel et al., "Intensity of vortices: from soap bubbles to hurricanes", Sci. Rep. 3, 3455 ; D0l:10.1038/ srep03455 (2013).

5• T. Meuel et al., "Hurricane track forecast cones from fluctuations", Sci. Rep. 2, 446 ; D0l:10.1038/ srep00446 (2012).

6• "How Bubbles could help measure hurricane intensity", Bulletin of the American Meteorological Society, News and Notes (avril 2014), p. 505.

Le présent article est partiellement basé sur le texte anglais suivant :

www.labnews.co.uk/features/soap-bubbles-in-a-spin/ 